\title{
Study on Product Innovative Design Process Driven by Ideal Solution
}

\author{
Fuying Zhang, Ximei Lu, Ping Wang, and Hui Liu \\ Tianjin University of Science \& Technology, \\ 300222 Tianjin, China \\ \{Fuying.Zhang, Ximei.Lu, Ping.Wang, Hui.Liu, zhfuying\} atust.edu.cn
}

\begin{abstract}
Product innovative design in companies today relies heavily on individual members' experience and creative ideation as well as their skills of integrating creativity and innovation tools with design methods agilely. Creative ideation and inventive ideas generation are two crucial stages in product innovative design process. Ideal solution is the desire final ideas for given problem, and the striving reaching target for product design. In this paper, a product innovative design process driven by ideal solution is proposed. This design process encourages designers to overcome their psychological inertia, to foster creativity in a systematic way for acquiring breakthrough creative and innovative solutions in a reducing sphere of solution-seeking, and results in effective product innovative design rapidly. A case study example is also presented to illustrate the effectiveness of the proposed design process.
\end{abstract}

Keywords: Ideal solution, innovative design process, contradiction resolving principles, theory of technical system evolution, function analysis.

\section{Introduction}

Product innovative design plays an important role for companies to win the competition in the market. In order to increase their innovation capacity, designers working in these companies must formalize their design procedures using tools and methods which will enable them to develop projects in the shortest possible time while guaranteeing that the solutions found will be innovative [1]. Creative ideation and creative ideas generation are two vital stages in product innovative design process. Creativity methods used in this stages such as brainstorming, morphological techniques, functional analysis encourage reflections from every angle and potentially lead people in a broad spectrum of directions generating a maximum number of ideas and select the best suitable ones to be implemented[2]. This makes the innovative design process inevitably have the risks of serial trials and errors and makes it difficult to develop innovative product in a short time. TRIZ[3] (Theory of Inventive Problem Solving), developed in Russia by G.S.Altshuller, provides systematic theories, methods and tools for product innovation design. The fundamental principle of TRIZ demonstrates that technological systems always tend to evolve in the direction of increasing ideality following a set of objective laws. The underlying problems product encountered are contradictions set by consumer and his desires with the objective laws which control 
evolution of product[4]. Solutions for such contradictions with technical means are invention which often results in product's progressing towards ideal state.

In this paper, the concept of ideal final result of TRIZ is first presented. Then the product innovative design process driven by IFR is proposed, which encourages designers to break through existing perception and overcome their psychological inertia; fosters their creativity to obtain breakthrough creative and innovative solutions in a reducing sphere of solution-seeking. Finally, a case study on hydrodynamic reciprocating seal is presented to illustrate the effectiveness of the proposed design process.

\section{The Concept of Ideal Final Result (IFR)}

Ideal Final Result (IFR) of TRIZ is the required or desired condition of an object. The ideal system performs a required function without actually existing. In practical, there's no absolute IFR, a product can be defined as ideal product when its functional performance improves, while its costs diminish. Ideal reflects the most utilization of system resources (both outside and inside). The more easily convertible the function resource, the more ideal the system is. The ideal level of the system can be denoted by the ideal degree $\mathrm{D}$.

$$
D=\frac{\sum F_{u}}{\sum F_{h}+\sum F_{c}}
$$

In this equation, useful function is represented by Fu; harmful function is represented by Fh; the cost relevant with function is represented by Fc.

Some general approaches can be used to increase the ideal degree D: (1) make the product have multifunctional; (2) make the product simplicity; (3) most make use of the system resources and environment to perform the product function; (4) eliminate the harmful function.

\section{Product Innovative Design Process Driven by IFR}

\subsection{The Problems Resolving Model Driven by IFR}

The essential of product innovative design is developing new product function and structure continuously to satisfy customer' new desires. TRIZ demonstrates that each product exists an ideal final result(IFR). All systems are developed towards ideal final result according to certain laws. Therefore, product innovative design is the process resolving conflicts which set by the consumer desires and the evolution laws that control these evolution.

The problems resolving model driven by IFR is shown in Fig.1. This model requires engineers to define the design problem correctly, precise formulate the functional ideal model, envision and state the ideal solution in relation to their projects at the beginning of design. Design problems is than presented in the shape of 


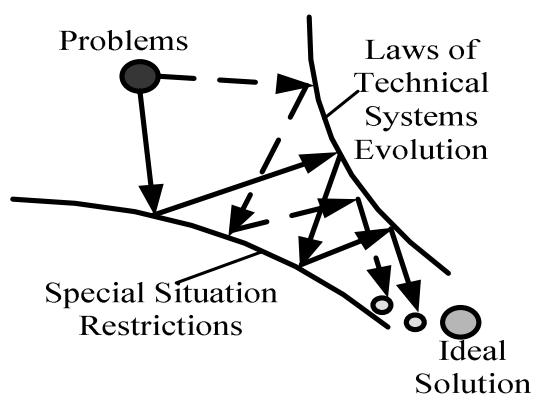

Fig. 1. Problems resolving model driven by IFR

a contradiction. Contradiction resolving principles are used to search the better ideal solution in accordance with the design restrictions and technology evolution laws. This model reduces the sphere of solution-seeking, encourages engineers to overcome their psychological inertia, provoke creative ideation and direct them towards the areas where effective solutions exist[5].

\subsection{The Product Innovative Design Process Driven by IFR}

According to above problems resolving model, the product innovative design process driven by IFR is proposed in Fig.2. The proposed design process comprises four essential stages: formulating ideal solution, problem analysis, problems resolving and identifying ideal solution. The formulating ideal solution stage is a formulate process for transforming the the initial situation such as design reqirements, the main components of system, character of interaction of the system's element, expressed in function, into ideal function that is the desired function. The problems analysis stage is a systemic analytic and modelate process for searching the problem space within the actual system, which is an underlying obstacle to hinder systems progression towards the ideal solution, for modelating the problems into TRIZ contradiction; The problem resolving stage is a eliminating problems process for resolving the defined problems in second stage using TRIZ tools to realize the ideal function; The identifying ideal solution stage is a synthetic evaluate process for searching the optimum ideal solution. Systematic creative methods such as TRIZ and function analysis are employed in the over all design process.

The formulation of ideal solution. The design process is begun by focusing on aims to be achieved rather than on detail of existing structure, or constraints and limitations on problems. The product and the given form and behavior of every one of its elements were designed to accomplish a predefined goal that customer desired[6]. In approaching this goal, the purpose and the accompanying physical action are important. They together constitute the function of an element. All the individual functional elements and the functional connections made by physical action constitute the function structure for product. 


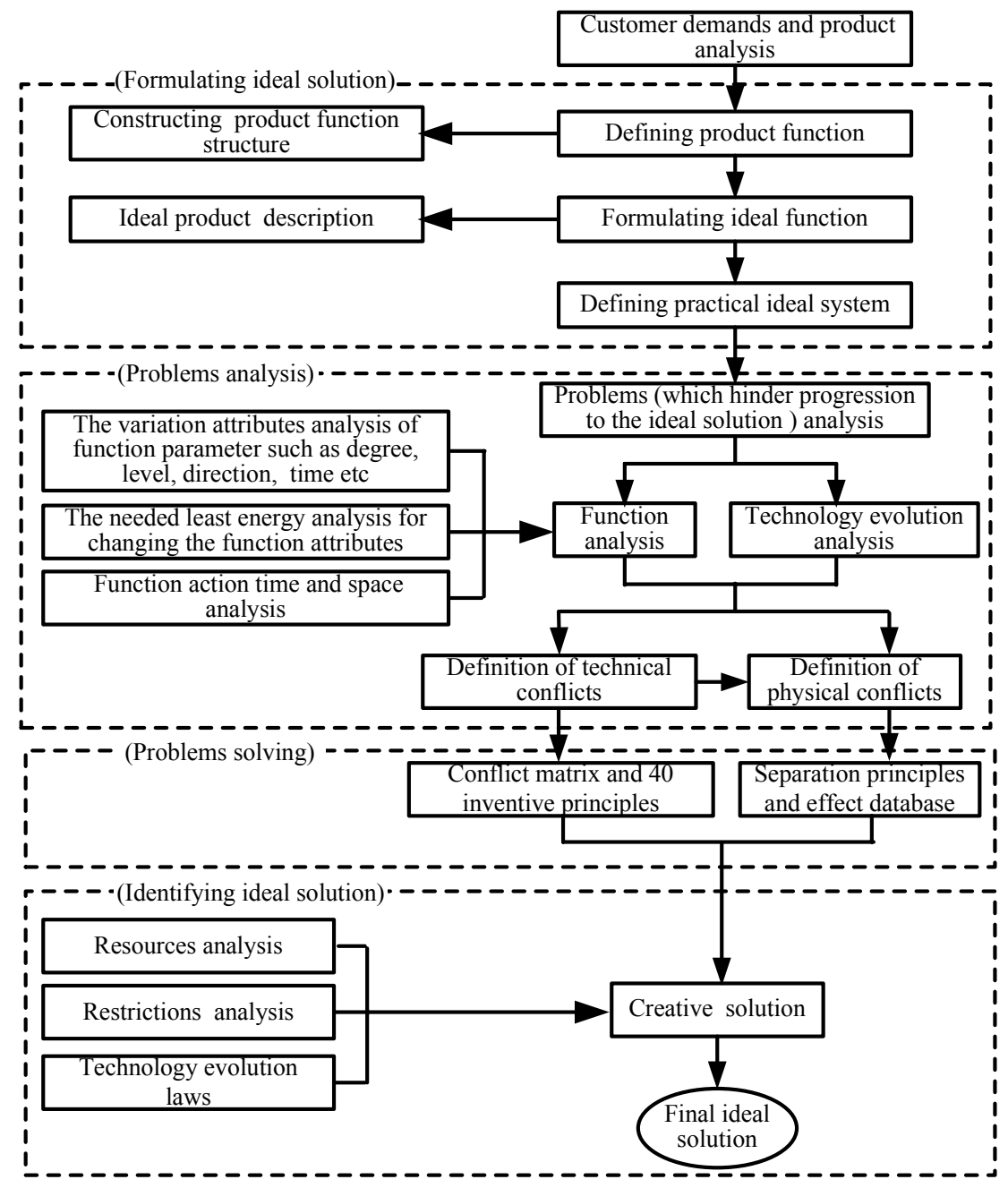

Fig. 2. Product innovative design process driven by IFR

After establishing the function structure, the ideal function state is examined, which successfully provides the required result of every component and then supplies the suitable conditions for performing it. For an practical ideal system, every function is performing at its best; the required result has been achieved.

During the process of defining ideal solution, one shoud approach the problem without any constraints and try to imagine the completely perfect ideal solution, by answering the problems such as "what are the desire product we want?" to discover the ideal solution. Ideal system enable us to find the path to a solution in a systematic way. 


\section{- Conflicts analysis}

In this stage, the conditions that have to be fulfilled in order to realize the ideal system are tried to find. This decision-making procedure may lead to contradiction within the actual system.

Looking from the ideal solution, all the functional elements should be in their complete functionlity. However, in reality there is always physical deficiency. According to the function structure built in the first stage, the useful, harmful, insufficient and redundancy functions are distinguished, the variational attributes for function parameters are analyzed, the action time and direction of function, the needed least energy for changing the function attribute and the ideal action time and space are identified. These specific, detailed and precise information help designer to find the physical deficiency in detail with respect to the physical parameters of the elements which result in the formulation of the conflict functions and conflict physical parameters.

Accordingly, each component in the function structure should be represented as a function state that provides the required result. The ideal system is analyzed from the base of our present scientific knowledge such as the technology evolution process, the evolution patterns related to product and its evolutional radar plot.

Through the evolution process analysis of product, The potential states of product that can led to the ideal function are identified, and the physical shortcoming in detail with respect to the physical cause that produced the unwanted physical phenomena are formulated.

Two sorts of contradictions can follow from the two aspects of the ideal system that govern the improvement and development of system component. The first aspect requires that the functional component possess the opposed attributes that ensure the complete performance of its required function. The second aspect requires that the component should not possess qualities that are not essential for its function. Two sorts of contradictions are formulated in the manner of TRIZ technical and physical conflict statement.

\section{- Conflicts resolving}

To eliminate the contradictions, identified in the above stage, TRIZ contradiction matrix and 40 inventive principles, separation principle and effect data are used. With the help of TRIZ analogical examples and effect data, the situation to a related situation can be connected with the same source of contradiction and the inventive ideas can be designed for a technical solution.

- Identifying the ideal solution

The inventive ideas can be examined and evaluated using the concept of ideal solution. The selected optimum solution should be outperforms in utilizing system resource, harness the harmful phenomenon to perform the benefit, and meeting the properties required in accordance with the restrictions and technology evolution laws.

The proposed innovative design process integrates the problem solving and solution selection process into problem analysis, which reduces the sphere of solutionseeking and makes the problem resolving quickly. 


\section{Case Study: Hydrodynamic Reciprocating Seal Innovative Design}

The hydrodynamic reciprocating seal is built upon the theories of lubrication, tribology, wear, heat transfer, material chreacteristics, and structure design of sealing interface. It is the most complex application situation and the most severe demand for dynamic seal in the hydraulic equipments[7]. In this paper, the reciprocating seal innovative design process is performed based on the product innovative design process driven by IFR.

- The analysis of problem space

The basic assembly of a $\mathrm{O}$ ring seal for a reciprocating piston of a hydraulic cylinder is shown in Fig.3. It is composed of moving piston, seal component installing in the annular sealing housing of piston, and cylinder. At rest, leakage gate is complete blocked by seal component between sealing interface. In case of alternating and reciprocating motions, together with the formation of lubricating film, the sealing interface separates a little because of the interaction of all kind of factors, and result in the shaping of leakage gate. If there is a flow of fluid in the leakage gate, it is called leak [8].

Market investigation determines the demands of customer for reciprocating seal. They are described as: longer service life, lower price, reliable sealing, small stickslip resistance, compact structure, easy to assemble.

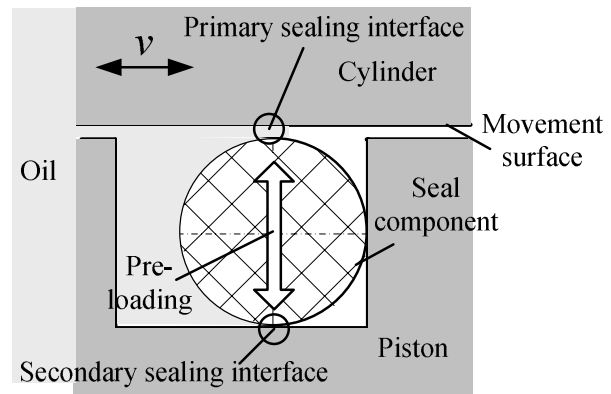

Fig. 3. Basic assembly of the seal

- Formulating the ideal solution of reciprocating seal

The function of hydrodynamic reciprocating sealing is to block the leakage gate while piston reciprocating motion. The function structure of reciprocating seal using seal component is showed in Fig.4. Seal components press cylinder to block leakage gate to perform seal function, the seal components will also increase the wear and friction between seal interface. Contact seal component has many good parameters, such as large pre-loading, lower elastic modulus, larger fracture tensile stretch, higher Poisson's ratio, lower shear modulus. But the essential parameter for best seal performance is the pre-loading. Increasing pre-loading improves the result, but brings a drawback that increases the wear and friction between seal interface. According to IFR concept of TRIZ, in the ideal state, the pre-loading of reciprocating seal will be maximum, and there is no wear and friction between reciprocating surface. 


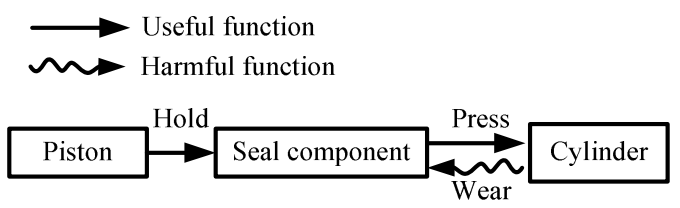

Fig. 4. Function constructure of reciprocating seal

- Problems analysis for reciprocating seal

In practice, high pre-loading of reciprocating seal component brings the serious friction and wear. To get high seal capability, the pre-loading has to be very high, but to decrease friction and wear, the pre-loading has to be low. A parametric conflict is revealed.

According to the function structure built in Fig.4, whether piston is at rest or during reciprocating movement, the system must perform the useful seal function. The harmful function (wear) appears only during reciprocating movement. The seal and wear spaces are the seal interface; favorable hydrodynamic lubrication is the primary measure for decreasing wear and friction.

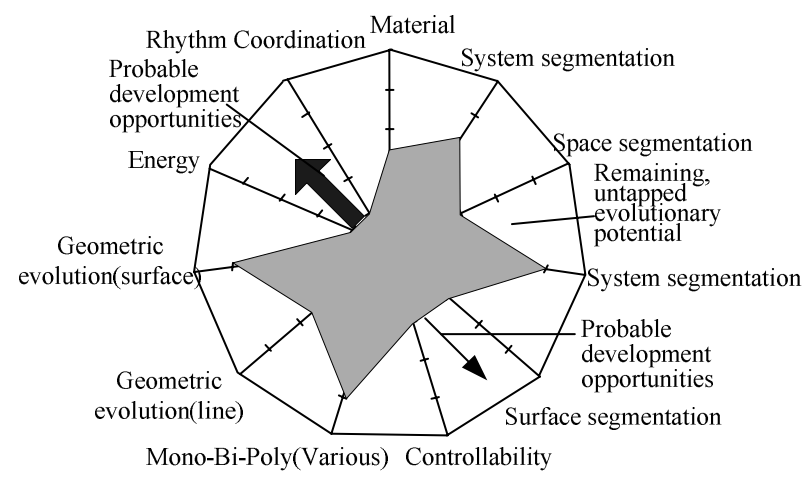

Fig. 5. Evolutionary potential plot of hydrodynamic reciprocating seal

Reciprocating seal problems are formulated with TRIZ conflict statements. Technical conflict is: when we improve the seal capablity, the lifecycle of seal element are deteriorated. Physical conflict is: pre-loading is needed high and low.

Based on the structrue evolution analysis for current reciprocating seal, paper [9] constructed the evolutionary potential plot of hydrodynamic reciprocating seal shown as Fig.5, and concluded this study as follows: in order to perform the desired function, it is necessary to improve the ability of adjustable of the seal component and to increase the segments of configuration, shape, highlight the utility of systems resource.

- The problems resolving for reciprocating seal

The technical conflict of reciprocating seal is formulated with standard features No.11 (stress or pressure), No.13 (stability of object's composition). According to TRIZ 
conflict matrix, four inventive principles are obtained: No.35 (parameter changes), No.2 (separation), No.33 (homogeneity), No.40 (composite materials). The opposed parameter demands are at the same component. But they are performed in different conditions. Separation principle in condition is use to solving the physical conflict properities of reciprocating seal. No.35 (parameter changes), No.33 (homogeneity) are the usable inventive principles. Fig.6 (a), (b), (c), (d) are the seal problems' solutions corresponding to the inventive principles of No.35, No.2, No.33, No.40.

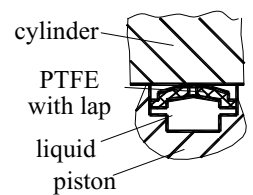

(a)

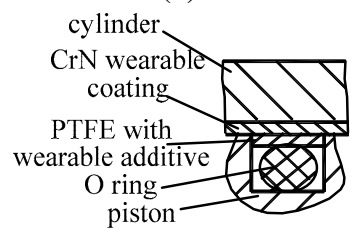

(c)

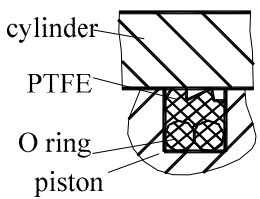

(b)

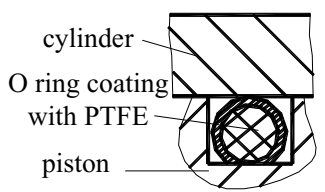

(d)

Fig. 6. Problem solving ideas for reciprocating sealing

- Identifying ideal solution for reciprocating seal

After the system resources analysis of reciprocating seal such as the shapes, materials, dimensions of seal component, cylinder and piston; the characteristics of oil in viscidity, flow, penetrate, pressure; the movement velocity of piston; considering the fluid pressure and temperature and relying on the possible direction pointed out by evolutionary potential plot of hydrodynamic reciprocating seal; the solution for reciprocating seal (shown in Fig.6 (a)) is selected as the final ideal solution. This solution can easily form hydrodynamic lubrication oil film by oil viscidity and the reciprocating movement velocity of piston so as to decrease the wear and frication. The characteristics of oil in flow, heavy, pressure and penetrate are used to increase the seal reliability. This reciprocating seal have the properties such as simple structure, small stick-slip resistance, good controllability and harmonic.

\section{Conclusion}

Innovative design plays an important role in new product development. In this paper, a innovative design process driven by IFR integrating with function analysis method and TRIZ theory is proposed, which is composed of formulating ideal solution, problem analysis, problem resolving and identifying ideal solution four stages. The essential of this design process is to encourage designers to overcome their psychological inertia and stress to get breakthrough innovative solutions, so as to achieve positive 
effect on the inventiveness of the product design rapidly in a reducing sphere of solution-seeking during their creative ideation and inventive ideas generation stages. The hydrodynamic reciprocating seal innovative design process is presented to illustrate the effectiveness of the proposed design process.

\section{Acknowledgments}

This research is sponsored by Natural Science Foundation of Tianjin (Grant No. 09JCYBJC05400) and Natural Science Foundation of China (Grant No. 50675155).

\section{References}

1. Cavallucci1, D.: Integrating Altshuller's development laws for technical systems into the design process. Annals of the CIRP 50, 115-120 (2001)

2. Hsiao, S.W., Chou, J.R.: A creativity-based design process for innovative product design. Industrial Ergonomics 34, 421-443 (2004)

3. Altschuller, G.S.: Creativity as an Exact Science. Gordon \& Breach, New York (1988)

4. Cavallucci, D., Lutz, P.: Converging in problem formulation: a different path in design. In: The Proceedings of DETC/DTM 2002 ASME Design Engineering Technical Conferences(2002)

5. Michael, A.O.: Inventive thinking through TRIZ: a practical guide. Springer, Heidelberg (2006)

6. Helfman, J.: The analytic inventive thinking model. Open University of Israel, Tel Aviv (1988)

7. Zhang, F., Xu, Y., Liu, H.: Seal technology study of hydrodynamic piston shaft based on TRIZ Su-field models and standard solutions. Lubrication Engineering 171, 57-60 (2005)

8. Mnler, H.K., Nau, B.S.: Fluid sealing technology-principles and applications. China Machine Press, Beijing (2002)

9. Zhang, F., Zhang, L., Wang, P.: Study of Product Innovative Design Based on TRIZ Evolution Theory. Transactions of the Chinese Society of Agricultural Machinery 39, 116-119 (2008) 